\title{
Analytical Modeling of Thermal Stresses in Plated Through Via (PTV) Structures
}

\author{
Jin Ma and Jan K. Spelt
}

\begin{abstract}
Plated through via (PTV) structures are widely used in printed circuit boards for interconnect. Due to the mismatch in the coefficient of thermal expansion (CTE) between the PTV and the board material, high thermal stresses can be induced in the PTV during high temperature soldering and normal usage. In particular, PTVs can fail due to cyclic temperature changes which cause thermal fatigue. This paper describes an analytical model of the thermal stresses in PTV structures using variational mechanics. Stress components are compared with those obtained using finite element analysis and with another analytical model.
\end{abstract}

Index Terms-Analytical model, plated through via (PTV), reliability, thermal stress, variational mechanics.

\section{INTRODUCTION}

A TYPICAL plated through via (PTV) structure consists of a cylindrical copper barrel, several pads, and the board material (FR4) as shown in Fig. 1. The difference in the coefficients of thermal expansion (CTE) of the copper and the FR4 causes thermal stresses in the structure. These thermal stresses are cycled due to temperature variation and can cause the PTV to fail due to fatigue. The magnitude of the stress depends on a number of factors, such as the barrel wall thickness, via diameter, board thickness, and material properties. New materials and designs are constantly being developed, most recently in response to the need to withstand the higher reflow temperatures associated with lead-free solders. From the designer's point of view, it is useful to have an analytical model to compute the thermal stresses as a function of geometric and material properties.

Due the complex geometry of the PTV structure, an exact analytical model is very difficult to build. Barker et al. [1] and Bhandarkar et al. [2] conducted finite element (FE) analyses of PTV structures. Recently, Fu et al. [3] conducted FE analysis of the PTV structures using McDowell's thermoplastic constitutive model. These FE results provided guidelines for the reliability design of PTVs; however, FE modeling and analysis are time-consuming, particularly when used to evaluate various design options and reliabilities. Oien [4] created a simple analytical model to compute the stress in the barrel. The barrel and surrounding FR4 were treated as two linear elements in parallel connection undergoing the same axial displacement when

Manuscript received October 2, 2003; revised September 22, 2004. This work was supported by the Center for Microelectronics Assembly and Packaging (CMAP).

J. Ma is with the School of Mechanical and Aerospace Engineering, Oklahoma State University, Stillwater, OK 74078 USA.

J. K. Spelt is with the Department of Mechanical and Industrial Engineering, University of Toronto, Toronto, ON M5S 3G8 Canada (e-mail: spelt@mie.utoronto.ca).

Digital Object Identifier 10.1109/TADVP.2005.848394

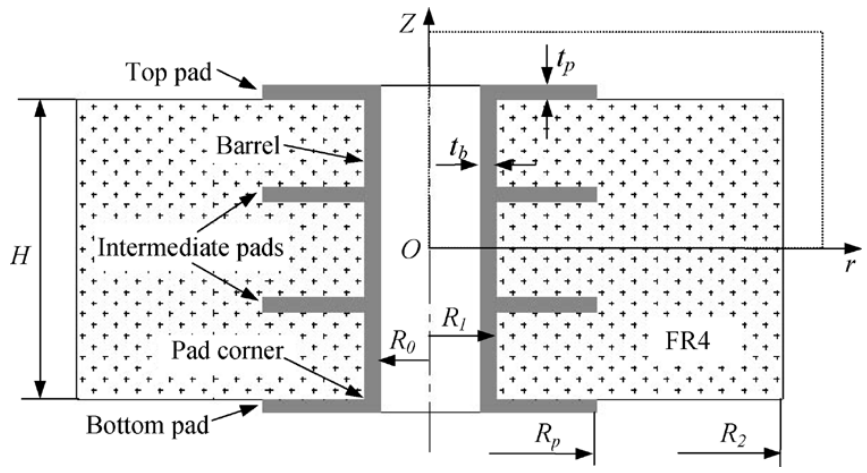

Fig. 1. Cross-sectional view of a PTV in a printed circuit board.

the temperature changed. Mirman [5] developed a more detailed model which took the pads into account. The pads, anchored on the barrel, constrain the axial expansion of the FR4. The pads were treated as thin circular plates, and their deflections were found based on the pressure against the FR4. The pads transfer the pressure to the barrel; hence, the axial stresses in the barrel could be found as well. The model did not include the effect of bonding between the barrel and the FR4. Since the drilled hole wall is cleaned and roughened before deposition of copper, and protruding glass fibers often provide additional anchoring of the copper to the FR4, both molecular bonding and mechanical bonding exist between the copper and the FR4. Neglecting this bond causes the barrel axial stress to be uniform between adjacent pads. The model of [5] also neglects the material outside the pad radius. FE studies have shown that the barrel stress decreases when the spacing is reduced [2], i.e., when less FR4 material is included in the model. The stress components other than the axial stress cannot be found from the analytical model of [5]; however, the interfacial shear stress may be important in determining debonding.

Whitney et al. [6] modeled PTV structures as three concentric cylinders, bonded at the interfaces. The inner cylinder was the copper barrel, the middle cylinder was the composite of the FR4 and the copper foil, and the outer cylinder was the FR4. Stress functions were found by assuming that the radial displacement was independent of board thickness, and that the axial displacement was proportional to the distance from the board midplane in the far field. The stress equilibrium condition was satisfied in each cylinder, but it was recognized that the model was incomplete because the compatibility condition was violated [6].

Modeling the PTV structures as concentric cylinders is an effective approach if proper stress functions can be found. Axisymmetry can be used to simplify the analysis since most PTV structures are axisymmetric. Of related interest, various studies 
of the thermal stresses and stress transfer between a reinforcing fiber and the matrix have been done for composite materials [7]-[9], where the fiber and the matrix are modeled as two bonded concentric cylinders. According to specific conditions, different assumptions of the stress functions have been made in order to derive the final stress equations. Nairn [9] analyzed the stresses around a fiber break. Since the fiber diameter is very small compared with other dimensions, it was assumed that the axial stress in the fiber was independent of radius. With this assumption, all the stress functions could be determined using variational mechanics to minimize the complementary strain energy [9].

In PTV structures, the wall thickness of the barrel is generally very small compared to its radius and height. Therefore, it is appropriate to assume that the axial stress in the barrel is independent of radius, i.e., uniform throughout the section. With proper boundary conditions, the thermal stresses in the PTV can be found using the approach taken with fiber-reinforced composites [9].

\section{ANALYTICAL MODELING}

\section{A. Stress Functions and Boundary Conditions for Two Concentric Cylinders}

The PTV structure is modeled as two concentric axisymmetric cylinders bonded at the interface $R_{1}$ as shown in Fig. 2 . The inner cylinder, made of plated copper, is isotropic, while the outer cylinder, consisting of the FR4 and the copper pads has different properties in the radial and axial directions. The determination of the outer cylinder composite properties and the range of $R_{2}$ will be discussed in later sections. The top and bottom pads can be treated as circular plates in bending. Due to symmetry in the $Z$ direction, only half of the cylinder needs to be modeled.

Due to the $\theta$ independence in axisymmetric stress states, two shear stress components, $\tau_{r \theta}$ and $\tau_{z \theta}$, are zero throughout the cylinders. The remaining four stress components are functions of $r$ and $z$. The stress equilibrium equations in the cylindrical coordinates are reduced to

$$
\frac{\partial \sigma_{r}}{\partial r}+\frac{\partial \tau_{r z}}{\partial z}+\frac{\sigma_{r}-\sigma_{\theta}}{r}=0 \text { and } \frac{\partial \tau_{r z}}{\partial r}+\frac{\partial \sigma_{z}}{\partial z}+\frac{\tau_{r z}}{r}=0 .
$$

Following [9], it is assumed that $\tau_{r z}$ and $\sigma_{r}$ can be expressed by two functions, $\Omega(r, z)$ and $\Psi(r, z)$, as

$$
\tau_{r z}=-\frac{\partial^{2} \Psi}{\partial r \partial z} \quad \text { and } \quad \sigma_{r}=\frac{1}{r} \frac{\partial \Omega}{\partial r}+\frac{\partial^{2} \Psi}{\partial z^{2}}
$$

From the stress equilibrium (1), $\sigma_{\theta}$ and $\sigma_{z}$ can be written as [9]

$$
\sigma_{\theta}=\frac{\partial^{2} \Omega}{\partial r^{2}}+\frac{\partial^{2} \Psi}{\partial z^{2}} \quad \text { and } \quad \sigma_{z}=\frac{\partial^{2} \Psi}{\partial r^{2}}+\frac{1}{r} \frac{\partial \Omega}{\partial r}
$$

To simplify the derivations and make the subsequent analytical derivations possible, it is assumed that the axial stress $\sigma_{z}$ in each cylinder is independent of $r$. This is justified since the wall thickness of the barrel $R_{1}-R_{0}$ is usually much smaller

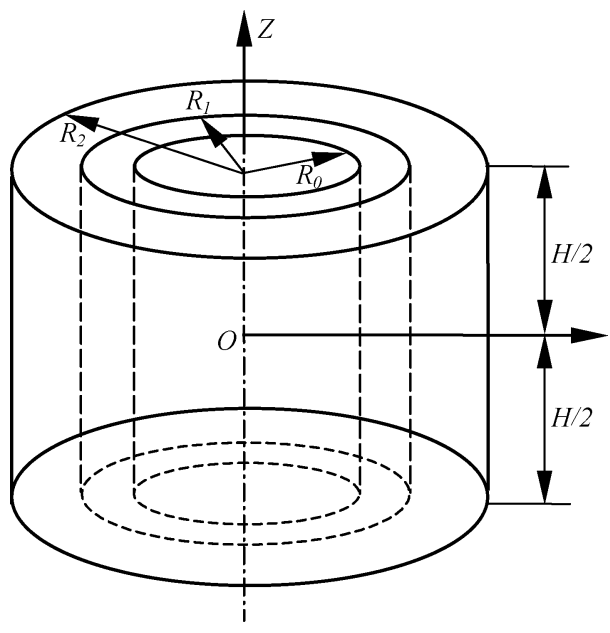

Fig. 2. Axisymmetric two-cylinder model for analytical modeling.

than $R_{1}$. If we choose $\Psi(r, z)$ as a combination of three arbitrary functions, $\psi_{0}(z), \psi_{1}(z)$, and $\psi_{2}(z)$, as

$$
\Psi(r, z)=\frac{1}{4} r^{2} \psi_{0}(z)+\psi_{1}(z) \ln r+\psi_{2}(z),
$$

and considering the radial compatibility equation

$$
\frac{\varepsilon_{r}-\varepsilon_{\theta}}{r}=\frac{\partial \varepsilon_{\theta}}{\partial r}
$$

it can be shown that the stresses in the inner and outer cylinders take the common form [9]

$$
\begin{aligned}
\sigma_{z} & =\psi_{0}(z) \\
\tau_{r z} & =-\frac{1}{2} r \psi_{0}^{\prime}-\frac{\psi_{1}^{\prime}}{r} \\
\sigma_{r} & =\frac{1}{16} r^{2} \psi_{0}^{\prime \prime}(3+v)+\frac{1}{4} \psi_{1}^{\prime \prime}[2(1+\nu) \ln r+(1-\nu)]+\psi_{2}-\frac{\psi_{4}}{r^{2}} \\
\sigma_{\theta} & =\frac{1}{16} r^{2} \psi_{0}^{\prime \prime}(3+v)+\frac{1}{4} \psi_{1}^{\prime \prime}[2(1+\nu) \ln r-(1-\nu)]+\psi_{2}+\frac{\psi_{4}}{r^{2}} .
\end{aligned}
$$

In the previous equations, $\psi_{2}(z)$ and $\psi_{4}(z)$ are redefined arbitrary functions of $z$ and all derivatives are with respect to $z$. The inner and outer cylinders may take different specific functions of $z$. The in-plane Poisson's ratio $v$ should be corresponding to each material.

For the convenience of description, we use subscript 1 for the inner cylinder and 2 for the outer cylinder. The stress boundary conditions for this problem are

$$
\begin{aligned}
\tau_{r z, 1}\left(R_{1}, z\right) & =0, \tau_{r z, 2}\left(R_{2}, z\right)=0 \\
\sigma_{r, 1}\left(R_{1}, z\right) & =0, \sigma_{r, 2}\left(R_{2}, z\right)=\sigma_{\text {edge }} \\
\sigma_{r, 1}\left(R_{1}, z\right) & =\sigma_{r, 2}\left(R_{1}, z\right) \\
V_{1} \sigma_{z, 1}(z) & =-V_{2} \sigma_{z, 2}(z) . \\
\sigma_{z, 1}\left(\frac{H}{2}\right) & =\sigma_{z, 2}\left(\frac{H}{2}\right)=0 \\
\tau_{r z, 1}\left(r, \frac{H}{2}\right) & =\tau_{r z, 2}\left(r, \frac{H}{2}\right)=0 \\
\tau_{r z, 1}(r, 0) & =\tau_{r z, 2}(r, 0)=0 .
\end{aligned}
$$


Equation (13) ensures the total force on any section is zero if we define the two area fractions as $V_{1}=\left(R_{1}^{2}-R_{0}^{2}\right) /\left(R_{2}^{2}-R_{0}^{2}\right)$ and $V_{2}=\left(R_{2}^{2}-R_{1}^{2}\right) /\left(R_{2}^{2}-R_{0}^{2}\right)$. It is noted that $V_{1}+V_{2} \equiv 1$ in all subsequent derivations. $\sigma_{\text {edge }}$ is a constant radial stress applied at the outer surface of the outer cylinder. Its effect will be discussed in later sections. Applying the boundary conditions (10) to (13), and considering that the constitutive relations and the radial displacements are equal at the interface, the functions $\psi_{1}(z), \psi_{2}(z)$ and $\psi_{4}(z)$ for each cylinder can all be expressed in terms of $\psi_{0}(z)$. Writing $\psi_{0}(z)=\psi(z)$, the stresses for the inner cylinder can be simplified as

$$
\begin{aligned}
\sigma_{z, 1} & =\psi(z) \\
\tau_{r z, 1} & =h_{1}(r) \cdot \psi^{\prime}(z) \\
\sigma_{r, 1} & =f_{1}(r) \cdot \psi^{\prime \prime}+f_{2}(r) G_{8} \cdot \psi+f_{2}(r) G_{9} \cdot T+f_{10}(r) \sigma_{\text {edge }}
\end{aligned}
$$

$\sigma_{\theta, 1}=f_{3}(r) \cdot \psi^{\prime \prime}+f_{4}(r) G_{8} \cdot \psi+f_{4}(r) G_{9} \cdot T+f_{11}(r) \sigma_{\text {edge }}$

and the stresses for the outer cylinder (composite of FR4 and pads) are

$$
\begin{aligned}
\sigma_{z, 2} & =-\frac{V_{1}}{V_{2}} \psi(z) \\
\tau_{r z, 2} & =h_{2}(r) \cdot \psi^{\prime}(z) \\
\sigma_{r, 2} & =f_{5}(r) \cdot \psi^{\prime \prime}+f_{6}(r) G_{8} \cdot \psi+f_{6}(r) G_{9} \cdot T+f_{12}(r) \sigma_{\text {edge }} \\
\sigma_{\theta, 2} & =f_{7}(r) \cdot \psi^{\prime \prime}+f_{8}(r) G_{8} \cdot \psi+f_{8}(r) G_{9} \cdot T+f_{13}(r) \sigma_{\text {edge }}
\end{aligned}
$$

In the aforementioned equations, $T$ denotes the temperature change, $G_{i}$ are constants in terms of material properties, and geometrical dimensions and are given in the Appendix . Functions $f_{i}(r)$ and $h_{i}(r)$ are defined in the Appendix as well. These constants and functions do not have any physical meaning explicitly. They are used to simplify the derivation. Once the geometry and material properties are specified, these constants and functions can be computed directly.

Nairn [9] pointed out that, due to the assumption that the axial stresses are independent of $r$, the axial stress would be wrong if the outer radius $R_{2}$ is larger than some critical value. To fix this problem, Nairn proposed using a third cylinder which has an infinite radius. The stress field in the third cylinder can be found from the above two cylinder models, by assuming infinite radius and length, hence, $\psi^{\prime}=\psi^{\prime \prime}=0$ and $\sigma_{\text {edge }}=0$. Following [9], the stresses in the third cylinder (FR4) are

$$
\sigma_{z, 3}=\tau_{r z, 3}=0 \quad \text { and } \quad \sigma_{r, 3}=-\sigma_{\theta, 3}=\frac{\sigma_{\infty}}{r^{2}}
$$

The expression for $\sigma_{\infty}$ is given in the Appendix. The effect of adding the third cylinder on the inside two cylinders can be accounted by simply setting $\sigma_{\text {edge }}=V_{1} \sigma_{\infty}$.

The stresses in all the cylinders are defined by one unknown function $\psi(z)$. Based on the principle of minimum complementary energy, the function $\psi(z)$ must minimize the total complementary energy in the three cylinder system. For computing the complementary energy, it is convenient to write the stresses in matrix form. The stresses in cylinder $i$ can be written as $\left[\sigma_{i}\right]=\left[B_{i}\right]\left[\psi_{i}\right]$, where $\left[\sigma_{i}\right]=\left[\sigma_{r, i}, \sigma_{\theta, i}, \sigma_{z, i}, \sigma_{r z, i}\right]^{T},\left[B_{i}\right]$ is a matrix for each cylinder, and $[\psi]=\left[T, \psi, \psi^{\prime}, \psi^{\prime \prime}, \sigma_{\infty}\right]^{T}$. For the inner cylinder

$$
\left[B_{1}\right]=\left[\begin{array}{ccccc}
f_{2}(r) G_{9} & f_{2}(r) G_{8} & 0 & f_{1}(r) & f_{10}(r) V_{1} \\
f_{4}(r) G_{9} & f_{4}(r) G_{8} & 0 & f_{3}(r) & f_{11}(r) V_{1} \\
0 & 1 & 0 & 0 & 0 \\
0 & 0 & h_{1}(r) & 0 & 0
\end{array}\right] .
$$

And for the middle and outer cylinders, the $[B]$ matrices can be written similarly.

The complementary energy $\Gamma$ can be expressed as (27) at the bottom of the page, where $R_{0 i}$ and $R_{1 i}$ denote the inner and outer radii of cylinder $i,\left[\alpha_{i}\right]=\left[\alpha_{r, i}, \alpha_{\theta, i}, \alpha_{z, i}, 0\right]$ is the thermal expansion coefficient tensor for cylinder $i$ in cylindrical coordinates. $\left[K_{i}\right]$ is the compliance tensor for cylinder $i$. It is a 4 by 4 symmetric matrix and can be found from the stress-strain constitutive relations. $\Gamma$ can be rewritten as

$$
\Gamma=\pi \int_{-H / 2}^{H / 2} d z\left([\psi]^{T}[C][\psi]+2 T[D][\psi]\right)
$$

where

$$
\begin{aligned}
& {[C]=\sum_{i=1}^{3} \int_{R_{0 i}}^{R_{1 i}} d r\left[B_{i}\right]^{T}\left[K_{i}\right]\left[B_{i}\right]} \\
& {[D]=\sum_{i=1}^{3} \int_{R_{0 i}}^{R_{1 i}} d r\left[\alpha_{i}\right]\left[B_{i}\right]}
\end{aligned}
$$

$[C]$ is a 5 by 5 symmetric matrix, and $[D]$ is a vector of length 5 . Performing the computations yields that $C_{13}=C_{23}=C_{34}=$ $C_{35}=D_{3}=0$. The integrand in (28) can be written as

$$
\begin{aligned}
2 T\left(D_{2}\right. & \left.+C_{12}+C_{25} \sigma_{\infty}\right) \psi+2\left(T D_{4}+T C_{14}+C_{45} \sigma_{\infty}\right) \psi^{\prime \prime} \\
& +2 C_{24} \psi \psi^{\prime \prime}+C_{22} \psi^{2}+C_{33} \psi^{\prime 2}+C_{44} \psi^{\prime \prime 2}+\lambda_{0}
\end{aligned}
$$

where $\lambda_{0}=\left(2 D_{1}+C_{11}\right) T^{2}+2 T\left(D_{5}+C_{15}\right) \sigma_{\infty}+C_{55} \sigma_{\infty}^{2}$ is independent of function $\psi(z)$.

Nairn [9] wrote the solution of $\psi(z)$ as

$$
\psi(z)=\varphi_{0}(1-\phi(z))
$$

$$
\Gamma=\sum_{i=1}^{3} \int_{0}^{2 \pi} d \theta \int_{R_{0 i}}^{R_{1 i}} d r \int_{-H / 2}^{H / 2} d z\left(\frac{1}{2}[\psi]^{T}\left[B_{i}\right]^{T}\left[K_{i}\right]\left[B_{i}\right][\psi]+T\left[\alpha_{i}\right]\left[B_{i}\right][\psi]\right),
$$


TABLE I

FR4 MATERIAL PROPERTIES [12]

\begin{tabular}{lcc}
\hline Property & Below $\mathrm{T}_{\mathrm{g}}=137^{\circ} \mathrm{C}$ & Above $\mathrm{T}_{\mathrm{g}}=137^{\circ} \mathrm{C}$ \\
\hline$E_{r \theta}=E_{r}=E_{\theta}(\mathrm{GPa})$ & 17.0 & 1.5 \\
\hline$E_{z}(\mathrm{GPa})$ & 7.4 & 1.0 \\
\hline$v_{r \theta}$ & 0.13 & 0.13 \\
\hline$v_{z}=v_{r z}=v_{\theta z}$ & 0.42 & 0.42 \\
\hline$G_{r \theta}=G_{r}=G_{\theta}(\mathrm{GPa})$ & 15.0 & 1.4 \\
\hline$G_{z}(\mathrm{GPa})$ & 2.4 & 1.5 \\
\hline$\alpha_{r \theta}=\alpha_{r}=\alpha_{\theta}(\mathrm{ppm})$ & 18.23 & 11.5 \\
\hline$\alpha_{z}(\mathrm{ppm})$ & 58.7 & 210 \\
\hline
\end{tabular}

where $\varphi_{0}$ is independent of $z$, and it can be found from a limiting case of this model. If the cylinders are long, the shear stresses far from the two ends will be zero, which implies that $\psi^{\prime}=\psi^{\prime \prime}=0$. Hence, $\psi$ is a constant, written as $\psi=\varphi_{0}$. Hence, in this case, the complementary energy $\Gamma$ is minimized when

$$
\varphi_{0}=-\frac{\left(D_{2}+C_{12}+C_{12} \sigma_{\infty}\right) T}{C_{22}} .
$$

The stresses near the top and bottom are found by minimizing $\Gamma$. The obtained Euler equation for $\psi(z)$ is

$$
\frac{d^{4} \psi}{d z^{4}}+p \frac{d^{2} \psi}{d z^{2}}+q \psi=q \varphi_{0}
$$

where $p=\left(2 C_{24}-C_{33}\right) / C_{44}$ and $q=C_{22} / C_{44}$. Obviously, $\varphi_{0}$ is a particular solution to (33). The final solution can be assumed to be $\psi(z)=\varphi_{0}(1-\phi(z))$, where $\phi(z)$ is the solution to

$$
\frac{d^{4} \phi}{d z^{4}}+p \frac{d^{2} \phi}{d z^{2}}+q \phi=0 .
$$

Boundary conditions (14) and (15) can be rewritten as

$$
\phi\left( \pm \frac{H}{2}\right)=1
$$

and

$$
\phi^{\prime}\left( \pm \frac{H}{2}\right)=0
$$

The solution can be found in the same way as illustrated in [9] and [10]. $\phi(z)$ can be written in closed form [(37) at the bottom of the page], where $\alpha_{1}=\sqrt{-(1 / 2) p+\sqrt{(1 / 4) p^{2}-q}}$, $\beta_{1}=\sqrt{-(1 / 2) p-\sqrt{(1 / 4) p^{2}-q}}, \alpha_{2}=(1 / 2) \sqrt{2 \sqrt{q}-p}$ and $\beta_{2}=(1 / 2) \sqrt{2 \sqrt{q}-p}$. Coefficients $A_{i j}$ are given in the Appendix. It is noted that the symmetry boundary condition (16) is satisfied in (37).

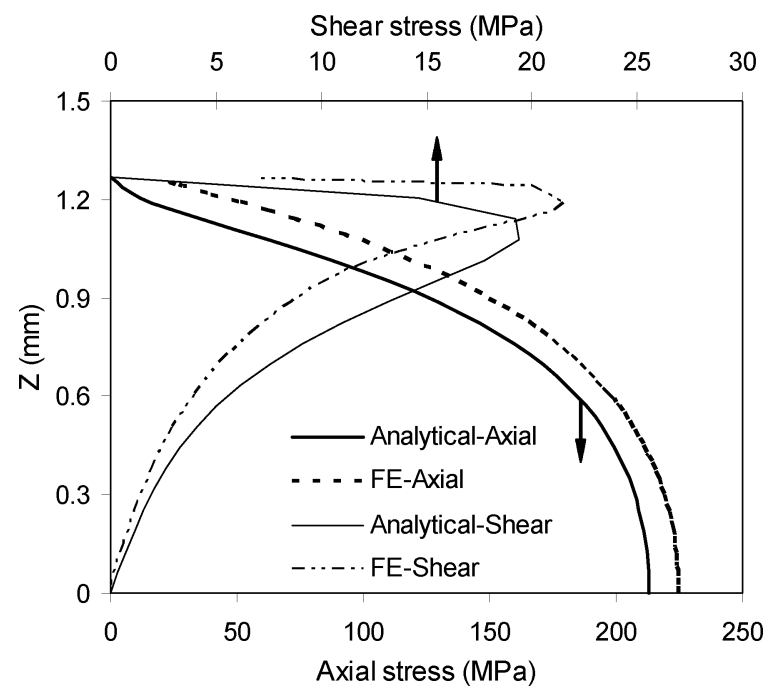

Fig. 3. Comparisons of axial stresses and shear stresses at $R_{1}$ in two concentric cylinders (Fig. 2 with dimensions $R_{0}=0.25 \mathrm{~mm}, R_{1}=0.30 \mathrm{~mm}$, $R_{2}=0.60 \mathrm{~mm}$, and $H=2.5 \mathrm{~mm}$ ) as a function of distance from the midplane $Z$.

So far, $\psi(z)$ has been found from (31), (32), and (37). All the stress functions for the two concentric cylinders can be found by plugging $\psi(z)$ and its derivatives into equations from (17) to (24).

\section{B. Results of Thermal Stresses in Two Concentric Cylinders}

In PTV structures, the inner cylinder is the barrel, made of isotropic plated copper, with Young's modulus taken as $E_{c}=$ $120 \mathrm{GPa}$, the Poisson's ratio $v_{c}=0.35$, and coefficient of thermal expansion $\alpha_{c}=17 \mathrm{ppm}$. The outer cylinder is the board, made of FR4 with typical properties as shown in Table I.

To verify the analytical model, an axisymmetric finite element model of two concentric cylinders was constructed with these same material properties and geometries. It was assumed that $R_{0}=0.25 \mathrm{~mm}, R_{1}=0.30 \mathrm{~mm}, R_{2}=0.60 \mathrm{~mm}$ and $H=2.5 \mathrm{~mm}$. It was further assumed that the temperature increased from $25^{\circ} \mathrm{C}$ to $125^{\circ} \mathrm{C}$, and there were no pads. The assumption that variation of the axial stress in the barrel in the radial direction is very small was confirmed by the FE model, except close to the ends (about twice the PTV wall thickness). Fig. 3 shows the comparison of the average axial stresses computed from FE analysis and the analytical model as a function of the distance from the midplane. The difference in axial stresses at the midplane, $Z=0$, is $5.4 \%$.

Shear stress at the bonded interface may be important in predicting debonding between the barrel and the FR4, especially when the FR4 surface is not well cleaned and preconditioned before plating. Fig. 3 also shows the comparison of shear stress

$$
\phi(z)=\left\{\begin{array}{cc}
A_{11} \cosh \left(\alpha_{1} z\right)+A_{12} \cosh \left(\beta_{1} z\right), & \left(0.25 p^{2} \geq q\right) \\
A_{21} \cosh \left(\alpha_{2} z\right) \cos \left(\beta_{2} z\right)+A_{22} \sinh \left(\alpha_{2} z\right) \sin \left(\beta_{2} z\right), & \left(0.25 p^{2}<q\right)
\end{array} .\right.
$$




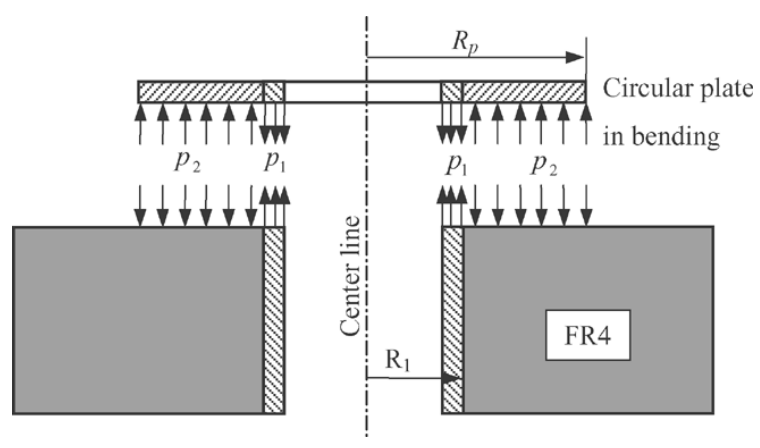

Fig. 4. Illustration of the top pad as an annular plate in bending.

$\tau_{r z}$ at the interface $R_{1}$. Both the FE and the analytical models show that the maximum shear stress occurs just below the board surface. The difference in predicted maximum shear stress is $10.7 \%$. It is noted that the stresses computed by the FE model do not satisfy the force surface boundary conditions strictly (Fig. 3) due to the approximations of the FE method. The axial stress in the barrel is dominant at the midplane, being five to ten times higher than the radial and hoop stress components.

\section{Treatment of External Pads in PTV Structures}

The coefficient of thermal expansion of the FR4 in the axial direction is generally 3 to 12 times greater than that of copper. Therefore, the FR4 (outer cylinder of Fig. 2) tends to expand more in the axial direction than does the barrel, and the copper pads on the top and bottom cause additional constraints to the FR4. To incorporate this effect, the top and bottom pads were modeled as annular plates clamped at $R_{1}$ as shown in Fig. 4. To simplify the derivation, it is assumed that the stress $p_{1}$ is constant at the junction with the barrel. If we write $p_{1}=\kappa \varphi_{0}$, where $0 \leq \kappa<1$ and $\kappa=0$ denotes there are no top and bottom pads, (35) can be written as

$$
\phi\left( \pm \frac{H}{2}\right)=1-\kappa
$$

From the force equilibrium of the two cylinders, $p_{2}=$ $-p_{1}\left(R_{1}^{2}-R_{0}^{2}\right) /\left(R_{p}^{2}-R_{1}^{2}\right)$ on the top surface of the FR4. With these assumptions, the displacement of the top surface of the FR4 and the pad can be written as

$$
W_{\text {pad }}(r, \kappa)=W_{c l}(r, \kappa)+W_{\text {shear }}(r, \kappa)
$$

where $W_{c l}(r, \kappa)$ is the classical solution to the deflection of a thin annular plate clamped at the inner radius under constant pressure, and $W_{\text {shear }}(r, \kappa)$ is the deflection due to shear deformation for thick plates. The deflection due to shear deformation cannot be neglected as the plate span to thickness ratio in the PTV structures can be as small as 3. From [13] and [14]

$$
\begin{aligned}
W_{c l}(r, \kappa)=\frac{1}{64} & \frac{p_{2}(\kappa) r^{4}}{D}+\frac{1}{4} C_{1} r^{2} \\
& +C_{2} \ln \left(\frac{r}{R_{p}}\right)+C_{3} r^{2} \ln \left(\frac{r}{R_{p}}\right)+C_{4}
\end{aligned}
$$

TABLE II

PARAMETERS FOR THE FE AND ANALYTICAL MODELS

\begin{tabular}{cccccccc}
\hline$R_{l}(\mathrm{~mm})$ & $R_{p}(\mathrm{~mm})$ & $R_{2}(\mathrm{~mm})$ & $t_{b}(\mathrm{~mm})$ & $t_{p}(\mathrm{~mm})$ & $H(\mathrm{~mm})$ & $T_{0}\left({ }^{\circ} \mathrm{C}\right)$ & $T_{\max }\left({ }^{\circ} \mathrm{C}\right)$ \\
\hline 0.3 & 0.48 & 0.96 & 0.05 & 0.05 & 2.5 & 25 & 200 \\
\hline
\end{tabular}

where $D=(1 / 12)\left(E_{c} t_{p}^{3} /\left(1-v_{c}^{2}\right)\right)$ is the bending rigidity and $t_{p}$ is the pad thickness. $C_{i}$ are constants and can be found from boundary conditions. From [15]

$$
\begin{aligned}
& W_{\text {shear }}(r, \kappa) \\
& =\frac{144 D\left(\left.\frac{\partial^{2} W_{c l}(r, \kappa)}{\partial r^{2}}\right|_{r=R_{1}}-\frac{1}{r} \frac{\partial W_{c l}(r, \kappa)}{\partial r}-\frac{\partial^{2} W_{c l}(r, \kappa)}{\partial r^{2}}\right)}{\pi^{4} G_{c} t_{p}}
\end{aligned}
$$

where $G_{C}$ is the shear modulus of the plate. $\kappa$ can be found by equating the area averaged deflection of the top surface of the FR4 and the area averaged deflection of the pad, i.e.,

$$
\int_{R_{1}}^{R_{p}} W_{\text {pad }}(r, \kappa) r d r=\int_{R_{1}}^{R_{p}} W_{\text {pad }}(r, \kappa) r d r
$$

The radial and tangential stresses in the pad due to bending are maximum at $R_{1}$. These two stresses can be computed as [13], [15]

$$
\begin{aligned}
\sigma_{r}^{\max } & =\frac{6 D}{t_{p}^{2}}\left(\frac{\partial^{2} W_{c l}(r, \kappa)}{\partial r^{2}}+\frac{\nu}{r} \frac{\partial W_{c l}(r, \kappa)}{\partial r}\right) \\
\sigma_{\theta}^{\max } & =\frac{6 D}{t_{p}^{2}}\left(\nu \frac{\partial W_{c l}(r, \kappa)}{\partial r}+\frac{1}{r} \frac{\partial^{2} W_{c l}(r, \kappa)}{\partial r^{2}}\right) .
\end{aligned}
$$

\section{Plasticity and Fatigue Life}

As an example, the present model can be used to estimate the elastic and plastic stresses and strains in PTV structures subject to cyclic thermal loads during manufacture and service. The largest thermal loads normally occur during soldering at temperatures that are usually between $180^{\circ} \mathrm{C}$ and $260^{\circ} \mathrm{C}$. The copper barrel may yield at these temperatures, creating compressive residual stresses after cooling which may enhance the service life in subsequent thermal fatigue.

The stress solutions derived in the previous section are all linearly dependent on the temperature change. This means that the stress can be calculated as a summation of the effect of each temperature increment during a thermal cycle. Under this circumstance, it is possible to estimate the plastic strain using the tangent modulus of copper after yielding. Plated copper usually shows some hardening after yielding [12]. In order to use the variational model directly to calculate the plastic strain and the new yield strength, the barrel can be assumed to yield uniformly [5]. For example, if the initial yield stress and the average plastic modulus are taken as $200 \mathrm{MPa}$ (at $0.2 \%$ strain) and $15.9 \mathrm{GPa}$ [12], respectively, the analytical model predicts that the barrel will have an average plastic strain of $0.4 \%$ at $200{ }^{\circ} \mathrm{C}$ for the PTV in Table II. This plastic strain would create a compressive residual axial stress of $190 \mathrm{MPa}$ in the barrel at $25^{\circ} \mathrm{C}$. This estimation is about $10 \%$ lower than that predicted by the 


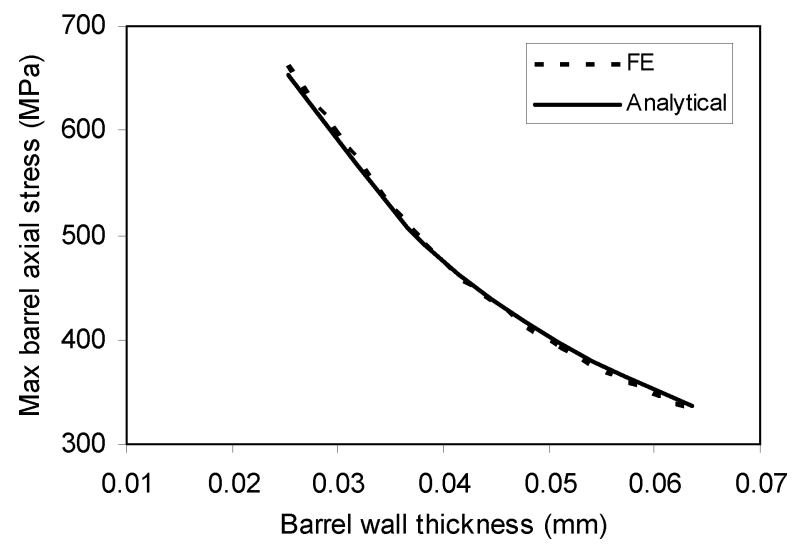

Fig. 5. Comparison of the maximum barrel stress as a function of the barrel thickness in PTV with external pads (Fig. 1, Tables I and II).

FE model. PTV structures usually first go through high-temperature reflows in manufacturing and relatively low temperature cycles in service or testing. A more detailed discussion of PTV plasticity incorporating both reflow and cycling is presented in the next section.

With the stress and strain calculated for manufacturing and in-service thermal cycles, the next step might be to use fatigue models to estimate the fatigue life of PTVs. If there is plastic deformation in each cycle for example, the service life could be estimated from the plastic strain range $\Delta \varepsilon_{p l}$ using the empirically established Coffin-Manson relationship [16]

$$
\Delta \varepsilon_{p l}=\varepsilon_{f}^{\prime}\left(2 N_{f}\right)^{c},
$$

where $\varepsilon_{f}^{\prime}$ and $c$ are fatigue ductility coefficient and exponent, respectively. Alternatively, the Basquin relation [17] might be used to compute the service life in terms of stress as follows:

$$
\frac{\Delta \sigma_{a}}{2}=\sigma_{f}^{\prime}\left(2 N_{f}\right)^{b}
$$

where $\Delta \sigma_{a}$ is the stress amplitude, and $\sigma_{f}^{\prime}$ and $b$ are the fatigue strength coefficient and exponent, respectively.

\section{RESUlTS FOR PTV STRUCTURES}

In order to verify the analytical model further, an axisymmetric FE model of a plated through via without intermediate pads was created to compute the stresses in the barrel and in the pads, as shown in Fig. 1. The material properties are shown in Table I. The dimensions and the temperature range are shown in Table II. Both the FE and the analytical models had the same parameters.

It was assumed that the PTV structure was stress free at $25^{\circ} \mathrm{C}$. Fig. 5 plots the barrel axial stresses at the midplane as a function of the barrel thickness, $t_{b}=R_{1}-R_{0}$, at $200{ }^{\circ} \mathrm{C}$. It can be seen that the predictions of the analytical model and the FE model are very close. It is noted that these stresses are greater than the yield stress of copper (approximately $200 \mathrm{MPa}$ ). Plastic deformation is considered later in the paper.

Lead-free solders are becoming more common, and consequently, soldering reflow temperatures are increasing. Fig. 6 shows that both models predict that the maximum barrel stress

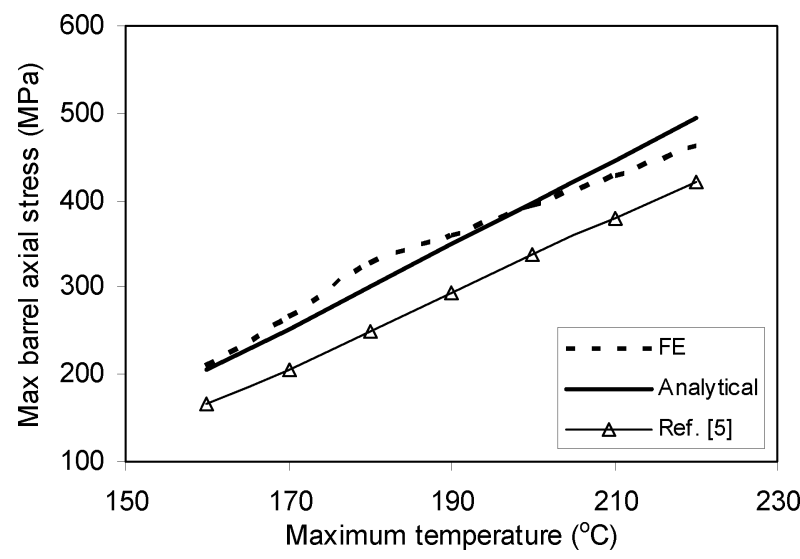

Fig. 6. Comparison of the maximum barrel stress as a function of the maximum temperature in PTV with external pads (Fig. 1, Tables I and II).

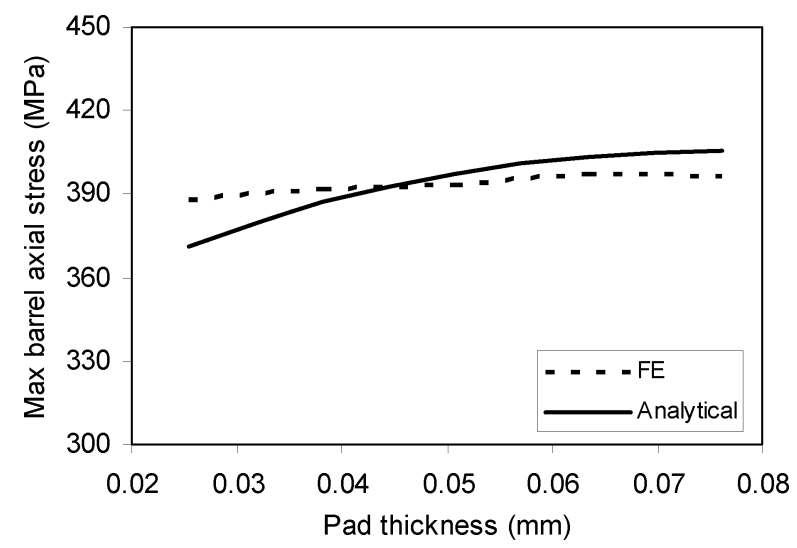

Fig. 7. Comparison of the maximum barrel axial stress as a function of external pad thickness (PTV of Fig. 1, Tables I and II).

increases with temperature approximately linearly. It is seen that an increase of maximum temperature from $170{ }^{\circ} \mathrm{C}$ to $220^{\circ} \mathrm{C}$ would double the barrel stress if conventional board material is used. A comparison with the result from [5] is given in Fig. 6. It is noted that the pad radius is the same as $R_{2}$ when using the model in [5]. However, the pad radius is as in Table II when using the model in this paper. It is seen that the prediction of the maximum barrel stress from [5] is $15 \%$ to $20 \%$ lower than the present model, mainly because the model from [5] assumed no bonding between the barrel and the FR4. It is noted that this assumption caused the stresses at the corner where the pad joins the barrel to be higher than in the present model, since it was assumed that all the loads are transferred to the barrel by the pads alone [5].

Pad thickness has a minor effect on the barrel stress at the midplane as shown in Fig. 7. The maximum difference between the FE and analytical models is $4.5 \%$. The present model predicts a slightly different trend because of the assumption that the stresses, $p_{1}$ and $p_{2}$, are uniform.

The aspect ratio (i.e., the ratio of the board thickness to the via outer diameter) can affect the maximum barrel stress. Fig. 8 shows that the barrel axial stress goes up as the board thickness increases. However, when the board thickness is more than eight times the via diameter, the barrel axial stress becomes less dependent on the board thickness, as the middle portion 


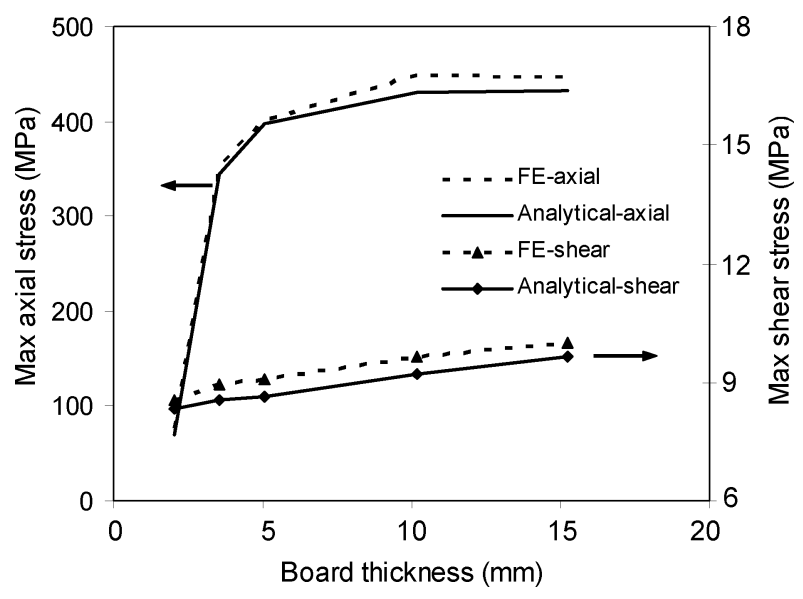

Fig. 8. Effect of board thickness on barrel stresses in PTV with external pads (Fig. 1, Tables I and II).

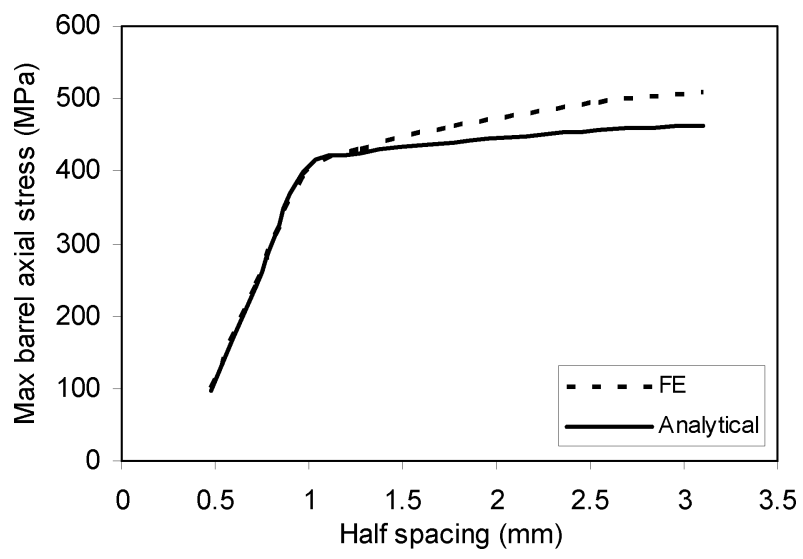

Fig. 9. Comparison of the maximum barrel stress as a function of $R_{2}$ in PTV with external pads (Fig. 1, Tables I and II).

of the barrel approaches the plane strain condition. The maximum shear stress, on the other hand, is almost independent of the board thickness.

The effect of an external pad can be seen by comparing Fig. 8 (with pad) and Fig. 3 (without pad), for the case that the board thickness is $2.5 \mathrm{~mm}(H=2.5 \mathrm{~mm})$. It is evident that the maximum axial stress has increased by more than $60 \%$ while the maximum shear stress has decreased by about $50 \%$ due to the external pad. The external pad decreases the shear stress, but causes greater elongation in the barrel.

The effect of spacing between two adjacent vias can be modeled analytically by equating $R_{2}$ with half of the distance between two via centers. The model of [5] cannot capture this feature well because it assumes that the outer cylinder extends only to the pad radius. Fig. 5 shows the effects of the spacing assuming that the outer cylinder surface is stress free. Both the FE and the analytical models show that the maximum barrel stress increases at two different rates as the spacing increases, depending on whether the spacing $2 R_{2}$ is smaller or greater than $2 \mathrm{~mm}$, given the other PTV parameters as in Table II. It is seen in Fig. 9 that the difference of the two predictions becomes larger as the outer radius $R_{2}$ increases. This is due to the assumption that the axial stress is independent of radius. However, when $R_{2}$ increases beyond $3 \mathrm{~mm}$, the stress approaches a constant. The

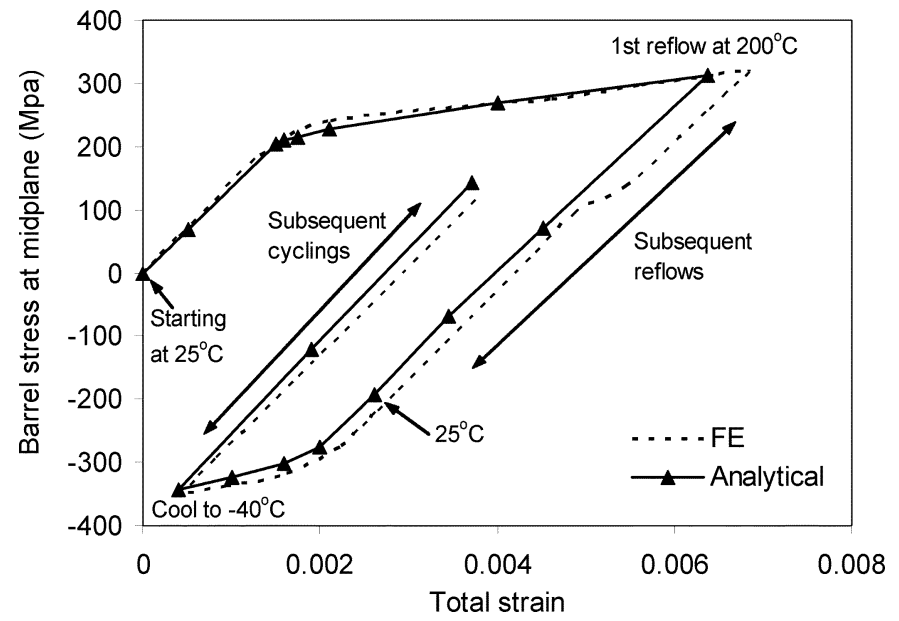

Fig. 10. Stress and strain at the barrel midplane due to reflow at $200^{\circ} \mathrm{C}$ and cycling between $-40^{\circ} \mathrm{C}$ and $125^{\circ} \mathrm{C}$ in PTV with external pads (Fig. 1, Tables I and II). The stress-free temperature of the copper barrel is $25^{\circ} \mathrm{C}$.

maximum difference between the FE and analytical models is about $10 \%$.

The stress-free temperature of PTVs can be chosen as the plating temperature, which is normally close to room temperature. The analytical model predicts that the PTV of Table II will have a new yield strength of about $300 \mathrm{MPa}$ due to hardening after undergoing a soldering process at $200^{\circ} \mathrm{C}$. No further plastic deformation will occur in subsequent soldering steps if the maximum temperature does not exceed $200{ }^{\circ} \mathrm{C}$. The analytical result agrees well with $\mathrm{FE}$ as seen in Fig. 10, with the maximum difference in the total strain being $8 \%$ at $200{ }^{\circ} \mathrm{C}$ for the PTV of Tables I and II. In accelerated thermal fatigue life testing of PTVs, the temperature range is often between $-40^{\circ} \mathrm{C}$ and $125^{\circ} \mathrm{C}$. As a further illustration of the use of the model, Fig. 10 also shows the stress and strain in the barrel during such fatigue cycling. The barrel remains elastic after the first cycle.

In normal service, because the maximum temperature will be less than the soldering temperature, no further plastic deformation will occur in barrels of nominal geometry, and the stress range can be used to estimate the thermal fatigue life using (46). In many real cases, however, the barrel may contain manufacturing defects due to imperfect drilling and plating. Such defects will create local stress concentrations which may increase the actual stresses to well beyond their nominal values. One possible approach to deal with this additional complexity would be to calculate the nominal stresses using the present analytical model and then apply stress concentration factors to estimate the maximum stresses.

\section{CONCLUSION AND Discussion}

An analytical thermomechanical model of two concentric cylinders, originally developed for composites, has been modified to compute the thermal stresses in PTV structures. This model assumes perfect bonding between the barrel and the FR4, and the axial stress in the barrel is independent of radius because the barrel wall thickness is small. The model uses variational mechanics to minimize the complementary energy and find the stress functions. The concentric cylinder model was extended to include the top and bottom pads by treating 
them as annular plates in bending. Plastic strain is estimated using the analytical model in the incremental form. Due to lack of other theoretical and experimental results, the results predicted by the variational model were compared with FE results extensively. Both results agreed well with each other.

FE analysis shows that the effect of intermediate pads on the maximum barrel stress is negligible if the pad is far from the midplane; e.g., the distance from the midplane is larger than the barrel diameter. If the pad is close to the midplane, the maximum axial barrel stress can be increased up to $10 \%$. This effect can be incorporated into the analytical model by changing the effective modulus and CTE with proper penalty functions.

When there are intermediate copper pads, the outer cylinder can be treated as a composite of the FR4 and the copper pads. This new composite material can still be treated as transversely isotropic because the in-plane mismatch of the copper and FR4 is small compared with the out-of-plane mismatch [18]. The effective in-plane modulus $E_{r \theta}^{\prime}$ and CTE $\alpha_{r \theta}^{\prime}$ can be found approximately using

$$
E_{r \theta}^{\prime}=\sum E_{i} V_{i} \text { and } \alpha_{r \theta}^{\prime}=\sum \alpha_{i} V_{i}
$$

where $V_{i}$ is the volume fraction of layer $i$. To account for the effects of different Poisson's ratios and interfaces, the effective in-plane modulus and CTE can be expressed as [18], [19]

$$
E_{r \theta}^{\prime}=\sum P_{i} E_{i} V_{i}, \alpha_{r \theta}^{\prime}=\frac{\sum Q_{i} \alpha_{i} E_{i} V_{i}}{E_{r \theta}^{\prime}}
$$

where $P_{i}$ and $Q_{i}$ are penalty functions for each material. The approximate effective out-of-plane CTE can be found from classical laminate theories using similar equations with different penalty functions.

Stress concentration is not considered in the model at the corner where the pad joins the barrel. This location can be more critical than the midplane if a stress concentration factor of 2 or 3 is considered. The actual stress concentration factor depends on the corner radius. Manufacturing defects in PTVs can create other sites of stress concentration that can dominate failure [20]. In this study, the barrel is assumed to be perfectly cylindrical. In real manufacturing, the geometry varies depending on the manufacturing quality. The stress can be different for irregular PTVs. However, from the design point of view, this model is sufficient to provide guidelines for the design of printed circit boards.

\section{APPENDIX}

DEFINED CONSTANTS AND FUNCTIONS

$$
\begin{aligned}
G_{1}= & \frac{1}{16} R_{0}^{2}\left[-1-3 \nu_{c}+4\left(1+\nu_{c}\right) \ln R_{0}\right] \\
G_{2}= & \frac{1}{16} \frac{V_{1}}{V_{2}} R_{2}^{2}\left[1+3 \nu_{r \theta}-4\left(1+\nu_{r \theta}\right) \ln R_{2}\right] \\
G_{3}= & \frac{1}{16} \frac{V_{1}}{V_{2}} R_{1}^{2}\left\{R_{1}^{2}\left(3+v_{r \theta}\right)-R_{2}^{2}\left[4\left(1+v_{r \theta}\right) \ln R_{1}\right.\right. \\
& \left.\left.+2\left(1-v_{r \theta}\right)\right]\right\}+\frac{1}{16} R_{1}^{2}\left\{R_{1}^{2}\left(3+v_{c}\right)\right. \\
& \left.-R_{0}^{2}\left[4\left(1+v_{c}\right) \ln R_{1}+2\left(1-v_{c}\right)\right]\right\}
\end{aligned}
$$

$$
\begin{aligned}
& G_{4}=\frac{G_{3}+\left(G_{1}-G_{2}\right) R_{1}^{2}}{1-\frac{R_{1}^{2}}{R_{0}^{2}}} \quad G_{5}=\frac{1-\frac{R_{1}^{2}}{R_{2}^{2}}}{1-\frac{R_{1}^{2}}{R_{0}^{2}}} \\
& G_{6}=E_{z} V_{2}\left\{E_{c} R_{0}^{2}\left[R_{1}^{2}\left(1-\nu_{r \theta}\right)+R_{2}^{2}\left(1+\nu_{r \theta}\right)\right]\right. \\
& \left.+E_{r \theta} R_{2}^{2} G_{5}\left[-R_{1}^{2}\left(1+v_{c}\right)+R_{0}^{2}\left(-1+v_{c}\right)\right]\right\} \\
& G_{7}=\frac{-1}{16} E_{c} E_{z} E_{r \theta} R_{0}^{2} R_{1}^{2} R_{2}^{2}\left\{\frac { V _ { 2 } } { E _ { c } } \left[\left(1+\nu_{c}^{2}\right)\left(R_{1}^{2}+4 R_{0}^{2} \ln R_{1}\right)\right.\right. \\
& \left.-2 R_{0}^{2}\left(1-\nu_{c}^{2}\right)-16\left(1-\nu_{c}\right)\left(\frac{G_{4}}{R_{0}^{2}}+G_{1}\right)\right] \\
& +\frac{V_{2}}{E_{r \theta}}\left[16\left(1-v_{r \theta}\right) G_{2}+\frac{V_{1}}{V_{2}} v_{r \theta}^{2}\left(R_{1}^{2}-4 R_{2}^{2} \ln R_{1}+2 R_{2}^{2}\right)\right. \\
& \left.\left.-\frac{V_{1}}{V_{2}}\left(R_{1}^{2}+4 R_{2}^{2} \ln R_{1}+2 R_{2}^{2}\right)\right]\right\} \\
& G_{8}=-R_{0}^{2} R_{1}^{2} R_{2}^{2} E_{r \theta}\left(E_{z} v_{c} V_{2}+E_{c} v_{z} V_{1}\right) \\
& G_{9}=R_{0}^{2} R_{1}^{2} R_{2}^{2} E_{c} E_{r \theta} E_{z} V_{2}\left(\alpha_{c}-\alpha_{r \theta}\right) \\
& G_{10}=R_{0}^{2} R_{1}^{2} R_{2}^{2} E_{z} V_{2}\left\{-E_{c}\left(R_{1}^{2}-R_{0}^{2}\right)\left(1-v_{r \theta}\right)\right. \\
& \left.+E_{x y}\left[R_{0}^{2}\left(1+v_{c}\right)+R_{1}^{2}\left(1-v_{c}\right)\right]\right\} \\
& G_{11}=\frac{E_{c} R_{2}^{2}}{2\left(1-v_{c}^{2}\right)}\left(\alpha_{r \theta}+\alpha_{z} v_{c}-\alpha_{c}-\alpha_{c} v_{c}\right) \\
& f_{1}(r)=\frac{1}{16} r^{2}\left(3+\nu_{c}\right)-\frac{1}{8} R_{0}^{2}\left[2\left(1+\nu_{c}\right) \ln r+1-\nu_{c}\right] \\
& +G_{1}+\left(G_{4}+\frac{G_{5} G_{7}}{G_{6}}\right)\left(\frac{1}{R_{0}^{2}}+\frac{1}{r^{2}}\right) \\
& f_{2}(r)=\frac{G_{5}}{G_{6}}\left(\frac{1}{R_{0}^{2}}-\frac{1}{r^{2}}\right) \\
& f_{3}(r)=\frac{1}{16} r^{2}\left(1+3 \nu_{c}\right)-\frac{1}{8} R_{0}^{2}\left[2\left(1+\nu_{c}\right) \ln r-1+\nu_{c}\right] \\
& +G_{1}+\left(G_{4}+\frac{G_{5} G_{7}}{G_{6}}\right)\left(\frac{1}{R_{0}^{2}}+\frac{1}{r^{2}}\right) \\
& f_{4}(r)=\frac{G_{5}}{G_{6}}\left(\frac{1}{R_{0}^{2}}+\frac{1}{r^{2}}\right) \\
& f_{5}(r)=\frac{1}{16} \frac{V_{1}}{V_{2}}\left\{-r^{2}\left(1+3 \nu_{r \theta}\right)+2 R_{2}^{2}\left[2\left(1+\nu_{r \theta}\right) \ln r+1-\nu_{r \theta}\right]\right\} \\
& +G_{2}+\frac{G_{7}}{G_{6}}\left(\frac{1}{R_{2}^{2}}-\frac{1}{r^{2}}\right) \\
& f_{6}(r)=\frac{1}{G_{6}}\left(\frac{1}{R_{2}^{2}}-\frac{1}{r^{2}}\right) \\
& f_{7}(r)=\frac{1}{16} \frac{V_{1}}{V_{2}}\left\{-r^{2}\left(1+3 \nu_{r \theta}\right)+2 R_{2}^{2}\left[2\left(1+\nu_{r \theta}\right) \ln r-1+\nu_{r \theta}\right]\right\} \\
& +G_{2}+\frac{G_{7}}{G_{6}}\left(\frac{1}{R_{2}^{2}}+\frac{1}{r^{2}}\right) \\
& f_{8}(r)=\frac{1}{G_{6}}\left(\frac{1}{R_{2}^{2}}+\frac{1}{r^{2}}\right) \\
& f_{10}(r)=\frac{G_{5} G_{10}}{G_{6}}\left(\frac{1}{R_{0}^{2}}-\frac{1}{r^{2}}\right)+\frac{R_{1}^{2}-\frac{R_{0}^{2} R_{1}^{2}}{r^{2}}}{R_{1}^{2}-R_{0}^{2}} \\
& f_{11}(r)=\frac{G_{5} G_{10}}{G_{6}}\left(\frac{1}{R_{0}^{2}}+\frac{1}{r^{2}}\right)+\frac{R_{1}^{2}+\frac{R_{0}^{2} R_{1}^{2}}{r^{2}}}{R_{1}^{2}-R_{0}^{2}} \\
& f_{12}(r)=1+\frac{G_{10}}{G_{6}}\left(\frac{1}{R_{2}^{2}}-\frac{1}{r^{2}}\right) \\
& f_{13}(r)=1+\frac{G_{10}}{G_{6}}\left(\frac{1}{R_{2}^{2}}+\frac{1}{r^{2}}\right)
\end{aligned}
$$




$$
\begin{aligned}
h_{1}(r)= & \frac{1}{2}\left(\frac{R_{0}^{2}}{r}-r\right), h_{2}(r)=\frac{1}{2} \frac{V_{1}}{V_{2}}\left(r-\frac{R_{2}^{2}}{r}\right), \sigma_{\infty}=G_{11} T \\
B_{11}= & \cosh \left(\frac{1}{2} \alpha_{1} H\right), B_{12}=\cosh \left(\frac{1}{2} \beta_{1} H\right) \\
B_{13}= & \alpha_{1} \sinh \left(\frac{1}{2} \alpha_{1} H\right), B_{14}=\beta_{1} \sinh \left(\frac{1}{2} \beta_{1} H\right) \\
A_{11}= & \frac{B_{14}}{B_{11} B_{14}-B_{13} B_{12}}, A_{12}=\frac{-B_{13}}{B_{11} B_{14}-B_{13} B_{12}} \\
B_{21}= & \cosh \left(\frac{1}{2} \alpha_{2} H\right) \cos \left(\frac{1}{2} \beta_{2} H\right) \quad \sinh \left(\frac{1}{2} \alpha_{2} H\right) \sin \left(\frac{1}{2} \beta_{2} H\right) \\
B_{22}= & \sinh \left(\frac{1}{2} \alpha_{2} H\right) \cos \left(\frac{1}{2} \beta_{2} H\right) \\
B_{23}= & \alpha_{2} \sin \left(\frac{1}{2} \beta_{2} H\right) \\
& -\beta_{2} \cosh \left(\frac{1}{2} \alpha_{2} H\right) \sin \left(\frac{1}{2} \beta_{2} H\right) \\
B_{24}= & \left.\alpha_{2} \cosh \left(\frac{1}{2} \alpha_{2} H\right) \sin \right) \\
& +\beta_{2} \sinh \left(\frac{1}{2} \alpha_{2} H\right) \cos \left(\frac{1}{2} \beta_{2} H\right) \\
A_{21}= & \frac{B_{24}}{B_{21} B_{24}-B_{23} B_{22}}, A_{22}=\frac{-B_{23}}{B_{21} B_{24}-B_{23} B_{22}} .
\end{aligned}
$$

\section{ACKNOWLEDGMENT}

The authors would like to thank Celestica Inc., Dy 4 Systems, and Alcatel Inc. for their generous support. The discussions with G. Parry of Coretec, Inc. and B. Birch of PWB Interconnect Solutions, Inc. and their suggestions are deeply appreciated.

\section{REFERENCES}

[1] D. Barker, M. Pecht, A. Dasgupta, and S. Naqvi, "Transient thermal stress analysis of a plated through hole subjected to wave soldering," J. Electron. Packag., vol. 113, pp. 149-155, Jun. 1991.

[2] S. Bhandarkar, A. Dasgupta, D. Barker, M. Pecht, and W. Engelmaier, "Influence of selected design variables on thermo-mechanical stress distributions in plated-through-hole structures," ASME J. Electron. Packag., vol. 114, pp. 8-13, Mar. 1992.

[3] C. Y. Fu, D. L. McDowell, and I. C. Ume, "Thermoplastic finite element analysis of unfilled plated-through holes during wave soldering," J. Electron. Packag., vol. 124, pp. 45-53, Mar. 2002.

[4] M. A. Oien, "Simple model for the thermo-mechanical deformations of plated-through-holes in multilayer printed wiring boards," in Proc. 14th Annu. Proc. Reliability Physics Symp., Apr. 1976, pp. 121-128.

[5] B. Mirman, "Mathematical model of a plated-through hole under a load induced by thermal mismatch," IEEE Trans. Compon., Hybrids, Manuf. Technol., vol. 11, no. 4, pp. 506-511, Dec. 1998.

[6] J. M. Whitney, I. U. Haq, and S. R. Soni, "Thermo-stress analysis of a plated-through-hole in a composite printed wiring board," J. Thermoplastic Composite Mater, vol. 6, pp. 29-37, 1993.

[7] L. N. McCartney, "New theoretical model of stress transfer between fiber and matrix in a uniaxially fiber-reinforced composite," Proc. $R$. Soc. Lond., vol. A425, pp. 215-244, 1989.
[8] J. A. Nairn, "On the use of shear-lag methods for analysis of stress transfer in unidirectional composites," Mechanics Mater, vol. 26, pp. 63-80, 1997.

[9] _ "A variational mechanics analysis of the stresses around breaks in embedded fibers," Mechanics Mater., vol. 13, pp. 131-154, 1992.

[10] Z. Hashin, "Analysis of cracked laminates: A variational approach," $\mathrm{Me}$ chanics Mater, vol. 4, pp. 121-136, 1985.

[11] J. N. Reddy, Energy Principles and Variational Methods in Applied Mechanics. New York: Wiley, 1984

[12] A. Dasgupta and V. Ramappan, "Simulation of the influence of manufacturing quality on reliability of vias," J. Electron. Packag., vol. 117, pp. 141-146, Jun. 1995.

[13] S. Timoshenko and S. Woinowsky-Krieger, Theory of Plates and Shells. New York: McGraw-Hill, 1959.

[14] R. Szilard, Theory and Analysis of Plates: Classical and Numerical Methods. Englewood Cliffs: Prentice-Hall, 1974.

[15] C. M. Wang, J. N. Reddy, and K. H. Lee, Shear Deformable Beams and Plates: Relationships with Classical Solutions. New York: Elsevier, 2000.

[16] O. Kraft, R. Schwaiger, and P. Wellner, "Fatigue in thin films: Lifetime and damage formation," Mater. Sci. Eng., vol. A319-321, pp. 919-923, 2001.

[17] S. Suresh, Fatigue of Materials, 2nd ed. Cambridge, U.K.: Cambridge Univ. Press, 1999, pp. 137-139.

[18] T. Y. Wu, Y. Guo, and W. T. Chen, "Thermal-mechanical strain characterization for printed wiring boards," IBM J. Res. Develop., vol. 37, no. 5, pp. 621-634, Sep. 1993.

[19] B. Hunter, G. Subbarayan, and D. Rose, "Characterization of PCB expansion using Moire interferometry and the impact of expansion variability on the life of solder joints," Advances in Electronic Packaging, vol. 26-1, pp. 875-882, 1999.

[20] M. A. Oien, "Methods for evaluating plated-through-hole reliability," in Proc. 14th Аnпu. Proc. Reliability Physics Symp., Apr. 1976, pp. 129-131.

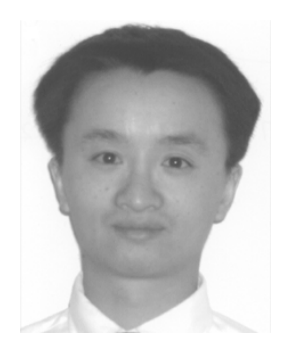

Jin Ma received the M.S. degree in mechanical engineering from Oklahoma State University, Stillwater, in 2002. He is currently pursuing the Ph.D. degree at Oklahoma State University.

His areas of interest include computational solid mechanics, mechanics of manufacturing processes, and nanoindentation.

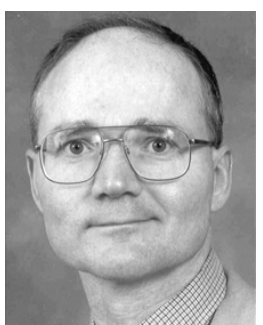

Jan K. Spelt received the Ph.D. degree in mechanical engineering from the University of Toronto, Toronto, ON, Canada, in 1985.

Currently, he is a Professor in the Department of Mechanical and Industrial Engineering, the University of Toronto. His research interests are in the areas of mechanics of materials and mechanics of manufacturing processes. In microelectronics packaging, past and present research projects include modeling of warpage in printed circuit boards during wave soldering and accelerated thermal fatigue testing and modeling of tin-lead and lead-free solder joints. 